\title{
Ostéonécroses des mâchoires induites par les bisphosphonates et pathologies osseuses bénignes : existe-t-il une association avec la polyarthrite rhumatoïde?
}

\section{Descroix V, Lescaille G, Coudert A, Javelot MJ, Cohen Solal M, Berdal A, Goudot P, Ruhin B (Service d'Odontologie, groupe hospitalier Pitié Salpétrière, Paris)}

Objectifs.

Les objectifs de cette étude étaient de recenser l'ensemble des cas d'ostéonécroses des maxillaires induit par la prise de bisphosphonates (ONMBP) chez des patients atteints de pathologies osseuses bénignes dans le but de décrire et comparer l'ensemble des caractéristiques de ces lésions chez les patients atteint d'ostéoporose et de polyarthrite rhumatoïde.

\section{Méthodes.}

L'ensemble des dossiers des patients adressés dans le service entre janvier 2007 et décembre 2011 pour le diagnostic, le traitement et le suivi d'ONMBP ont été analysés de manière rétrospective. Seuls les patients atteints de pathologies osseuses malignes ont été exclus de cette étude. L'ensemble des données démographiques, médicales et bucco-dentaires a été recherché ainsi que les éléments caractéristiques des lésions d'ONMBP (localisation, stade, facteurs déclenchant, traitements et suivi).

Résultats.

Durant une période de cinq ans, 112 patients ont été diagnostiqués pour une ONMBP dans notre service. Au sein de cette cohorte, 15 patients avaient reçus un traitement par BP pour le traitement d'une pathologie osseuse bénigne (âge moyen 65,7 $\pm 19,8$ ans, $80 \%$ de femmes). Ces patients ont reçu des BP pour des indications variées : 8 patients (53\%) pour prévenir une ostéoporose associée à une polyarthrite rhumatoïde, 6 (40 \%) pour prévenir les complications d'une ostéoporose idiopathique et 1 patient $(7 \%)$ pour une algoneurodystrophie de la cheville. La moyenne de temps d'exposition aux BPs était de 48,4 mois (médiane, 36 mois). Dans 13 cas $(86,6 \%)$ l'ONMBP a été diagnostiquée à la suite d'une extraction dentaire. Une chirurgie importante, alvéolectomie ou séquestrectomie, a dû être réalisée chez 9 patients (60\%), avec une cicatrisation qui a été obtenue pour l'ensemble des 15 patients entre 3 et 36 mois (moyenne 11,5 mois). L'analyse comparée des caractéristiques cliniques de l'ensemble des lésions d'ONM BP n'a pas mis en évidence de différence statistiquement significative entre les ONMBP des patients ostéoporotiques et de ceux atteints de polyartrite rhumatoïde.

Conclusions.

L'ostéonécrose des mâchoires est un effet indésirable rare des traitements par bisphosphonates et tout particulièrement lorsque ceux-ci sont administrés par voie orale. Dans les limites de notre étude nous n'avons pas pu mettre en évidence de différence significative entre l'aspect clinique, l'évolution, le traitement ou la résolution des lésions d'ONM chez les patients atteints de polyartrite rhumatoïde et les autres.

DESCROIX Vianney

vdescroix@gmail.com 